\title{
Psoriasis Severity Assessment of 2-D Psoriasis Skin Images
}

\author{
${ }^{1}$ S.Raaghavi, ${ }^{2}$ M.Ragini \\ ${ }^{1}$ Wipro Limited, United States of America, ${ }^{2}$ Nagoya Institute of Technology, Japan \\ raaghavi.teens@gmail.com, raginimanivannan@yahoo.com
}

\begin{abstract}
Psoriasis, meaning "itchy condition", is a chronic skin disease that is characterized by scaly, reddened patches. It is a recurring disease with varying severity ranging from slight limited flakes to entire body. Psoriasis Area and Severity Index (PASI) is the most conventional method for measuring the severity of this disease. It computes the PASI score, which ranges from 0 to 72, by combining the severity of lesions and area affected into a single computational score. But these scores are not reliable as they vary for the same psoriatic lesion among different physicians and suffer from inter- and intra-observer difference. This paper mainly focuses on assessing the severity index of 2D digital images of psoriasis by removing erythema (redness) from the selected image, thereby considering other skin cells for analysis. It makes use of "Feature Space Scaling" algorithm that relies on color contrast and image texture along with a combination of Support Vector Machine (SVM) classification filters and Markov Random Fields (MRF) to come up with a treatment solution. This algorithm is tested on different psoriasis affected skin images under various lighting conditions and is proved to be reliable.
\end{abstract}

Index Terms - Feature extraction, Markov Random Field (MRF), Support Vector Machine (SVM), Gabor filter, segmentation.

\section{INTRODUCTION}

$\mathrm{P}$ soriasis is a chronic inflammatory disorder of the skin that can affect a person at any age. It is characterized by abnormally excessive and rapid growth of epidermal layer of the skin. The causes of psoriasis are not fully understood. It is generally considered a genetic disorder, which can be triggered or influenced by environmental factors. There is no cure to psoriasis, but various treatments can control its symptoms. The identification of psoriasis is usually based on the appearance of skin. There are no special blood tests or diagnostic procedures for confirming the presence of psoriasis. The Psoriasis Area Severity Index (PASI) is the most widely used measurement tool to assess the severity of psoriasis. It combines the assessment of the severity of psoriatic lesions and the area affected into a single score. The severity is estimated by three clinical signs: erythema (redness), induration (thickness) and desquamation (scaling).

The algorithm presented in this paper provides automatic scaling and segmentation of digital images of psoriatic lesions.
The combination of Markov Random Fields (MRF) and Support Vector Machine (SVM) used in evaluation overcomes

the scaling segmentation problems such as variations in lighting conditions, skin type and types of psoriatic lesions. It also solves the binary classification problem by removing erythema from the affected area and it visualizes the remaining pixels either as normal skin or scaled skin. This classification of skin pixels is derived from the colour contrast between scaling and erythema and the roughness or texture of skin is analysed using a bank of Gabor filters.

\section{RELATED WORK}

\section{A. Computer Aided Psoriasis Continuous Area And Severity Score}

Though there are many automated techniques for the measurement of psoriasis, they are not suitable for trials in large scale. Hence a combination of the advantage of accurate computerized measurement of the affected area on the photographic image is combined with the physician's proficiency for the edge detection of the psoriatic lesion. In this method, the patients are examined and photographed before and after the treatment with calcipotriol ointment or placebo. The affected psoriatic area is outlined on the photograph manually by the physician and that area is automatically measured by a computer. For comparison, the physicians also carried out the standard psoriatic area assessment. The computer-aided measurement of the psoriatic lesion area tends to overestimate. The adapted PASI index, where the psoriatic area was not converted into an area grade, but was maintained as a continuous variable, also improved the power of the clinical trials. The modified PASI that involves computer-aided assessment as a continuous variable is named Computer aided psoriasis continuous area and severity score (cPcASI).

\section{SCALing Segmentation OF Psoriasis Skin IMAGeS}

The feature space algorithm uses both color and texture to classify the pixels. In some cases, the color of scaling is similar to that of the normal skin. So the rough texture of scaling is differently marked from normal skin. The three major phases involved in this algorithm are: 1) Feature extraction and object segmentation, 2) Pixel classification using SVM and MRF, 3) Detection of vague scaling and 4) 
Growth rate estimation and classification.

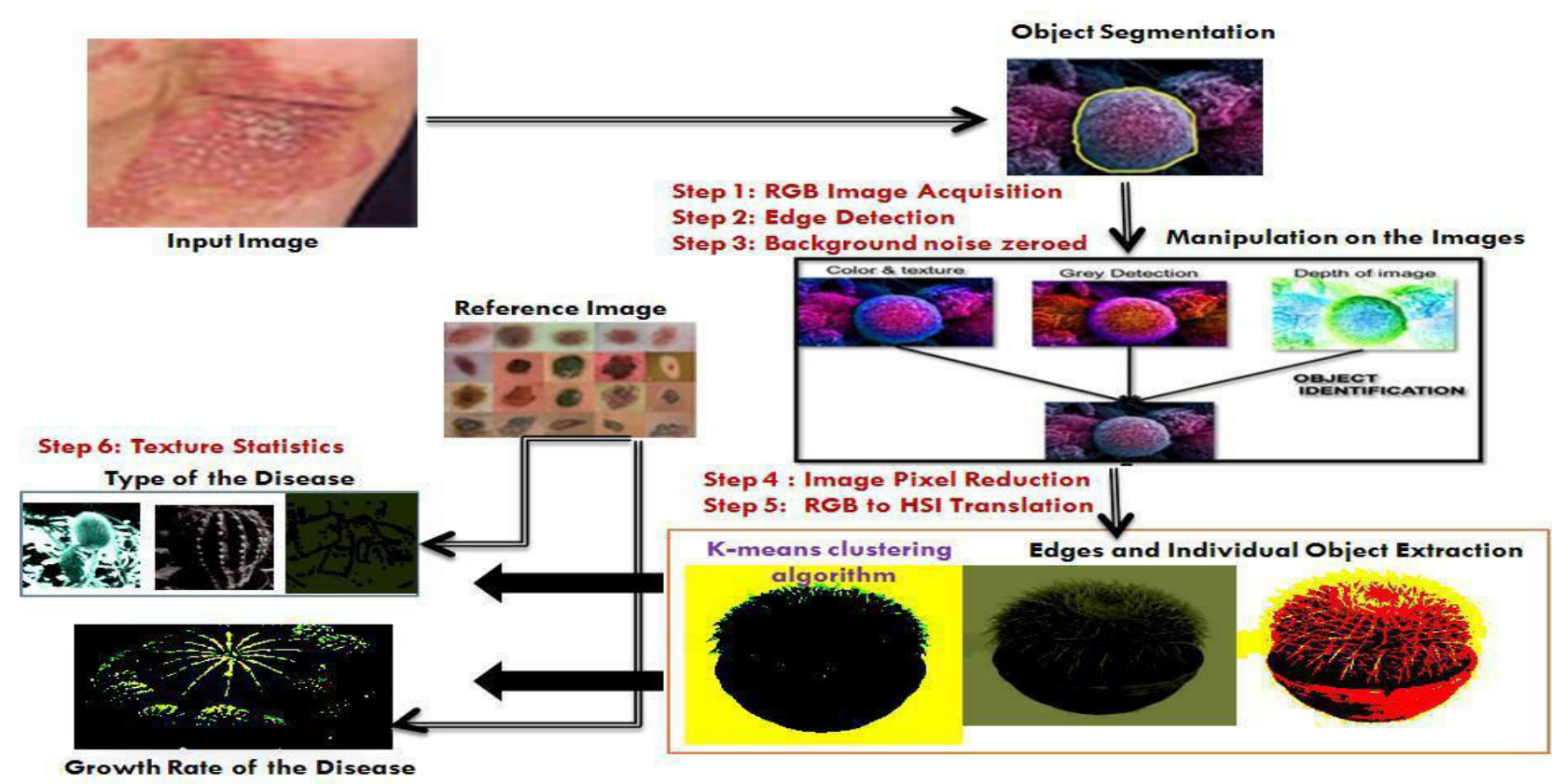

Fig. 1. Architecture diagram
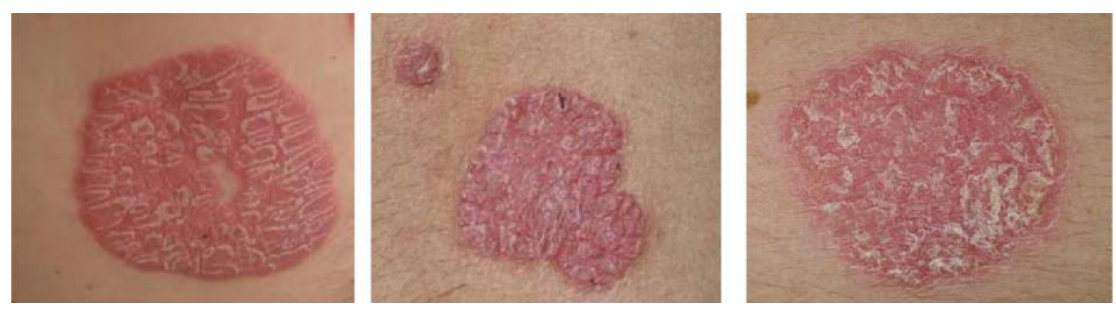

Fig.2 Examples for psoriasis images

\section{A. Feature Extraction And Object Segmentation}

The algorithm first analyzes skin color and then the texture is analyzed using a bank of Gabor filters to create a feature space for the image. It removes the erythema pixels and creates the training sets for the classification process.
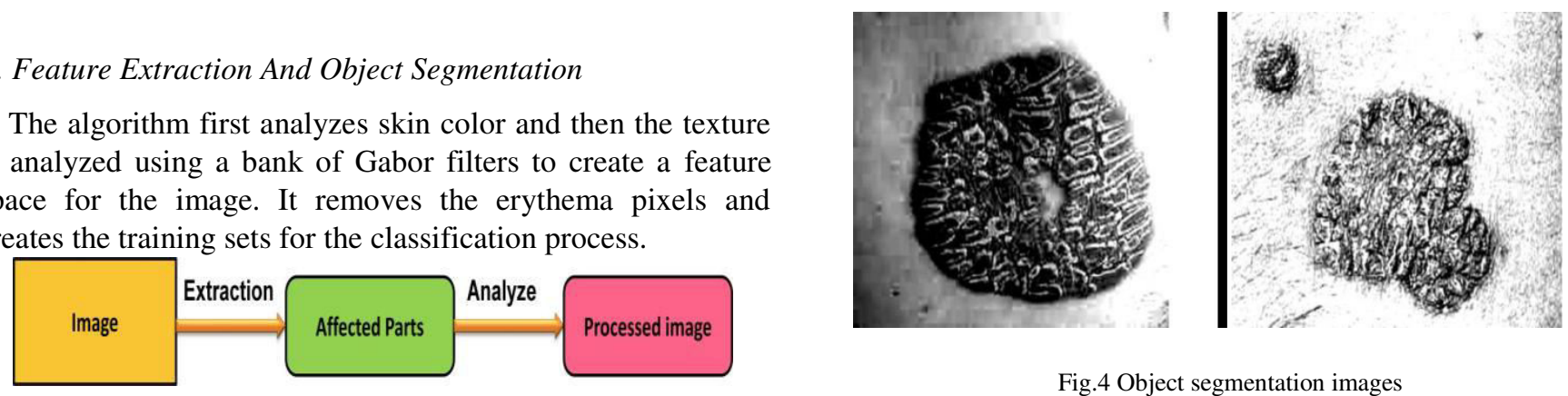

Fig.4 Object segmentation images

Fig..3 Feature extraction

In general, features are said to be a distinctive attribute or aspect of any object. A specific image feature, defined in terms of a specific structure in the image data, can often be represented in different ways. Extractions are a way to separate a desired substance when it is mixed with others. Here the goal in image analysis is to extract useful information for solving application-based problems. The input image is extracted with its features on order to validate in further processes.

\section{B. Pixel Classification Using SVM And MRF}

A Support Vector Machine (SVM) performs classification by constructing an $\mathrm{N}$-dimensional hyper plane that optimally separates the data. Markov random field (MRF), Markov network or undirected graphical model is a set of random variables having a Markov property described by an undirected graph. A pattern (object) is any item whose important characteristics form a set of descriptors. A descriptor is a variable (usually numerical) that characterizes an object. A descriptor can be any experimentally measured or theoretically computed quantity that describes the structure of a pattern: (biological samples). The input image is validated with SVM and MRF methodology to identify the nuances of the samples taken under study. 


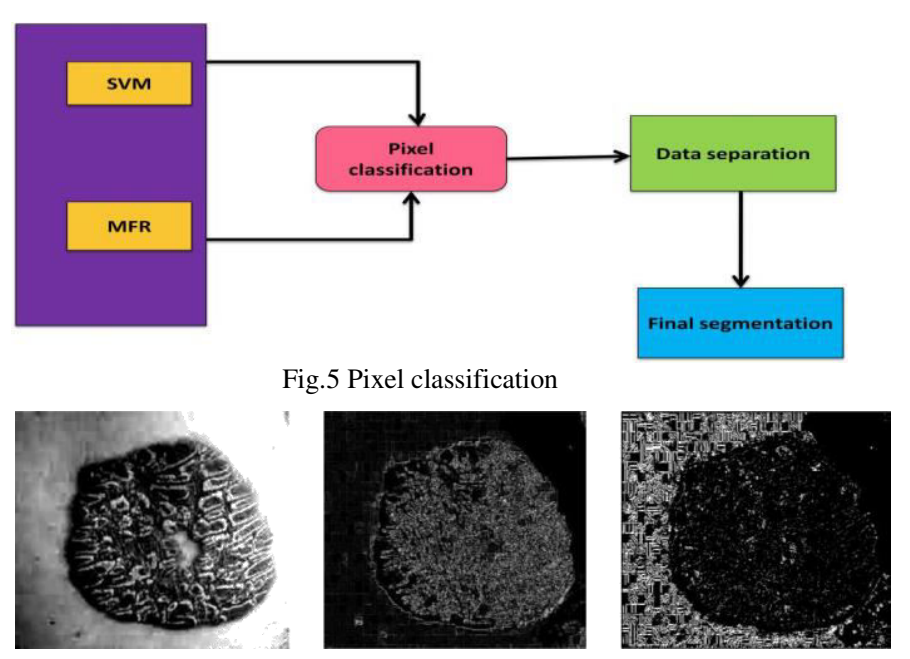

Fig. .6 a) SVM Segmentation b) MRF Segmentation c) Final Segmentation

We then make use of the soft constrained K-means clustering algorithm to obtain the training sets for the evaluation of psoriasis from the input image. The training set collected from a variety of skin images does not provide a good classification result. Therefore we obtain this by gathering directly from the input image being processed. This algorithm forms two clusters, one for scaling and other for skin pixels that is obtained by combining the classification results obtained by using the SVM.

\section{K-Means Clustering Algorithm}

The procedure follows a simple and an easy way to classify a given data set through a certain number of clusters, assume $\mathrm{k}$ clusters, with a fixed priority.

$$
J=\sum_{j=1}^{k} \sum_{i=1}^{n}\left\|x_{i}^{j}-c_{j}\right\|^{2}
$$

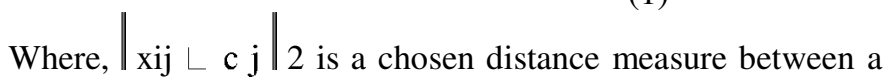
data point and the cluster centre is an indicator of the distance of the $\mathrm{n}$ data points from their respective cluster centres.

- Place $\mathrm{K}$ points into the space represented by the objects that are being clustered. These points represent the initial group centroids.

- Assign each object to the group that has the closest centroid. When all objects have been assigned, recalculate the positions of the $\mathrm{K}$ centroids.

- Repeat Steps 2 and 3 until the centroids no longer move. This produces a separation of the objects into groups from which the metric to be minimized can be calculated.

D. Temporal Pattern Matching Algorithm

$$
\left(\begin{array}{l}
n \\
k
\end{array}\right)=\left(\begin{array}{c}
n+k-1 \\
n-1
\end{array}\right)=\left(\begin{array}{c}
n+k-1 \\
k
\end{array}\right)
$$

(2)

It contains set of arrays, where each array contains all events of the same type, sorted by time stamps. Particular item in the pattern uses a binary search over the appropriate arrays. Considerably this is more expensive per item but it allows TPS to skip many unnecessary events in personal histories.

\section{E. Detection Of Vague Scaling}

The Scale Image command enlarges or reduces the physical size of the image by changing the number of pixels it contains. It changes the size of the contents of the image and resizes the canvas accordingly. The validated image is detected with its scaling range to identify the pixel transformation and intensity. This strategy evaluates the occurrence of the disease information at the particular portion or region in the sample image taken under study.

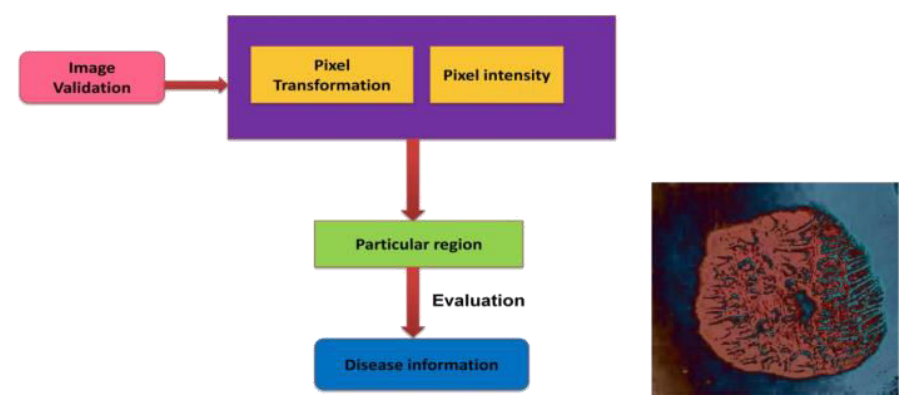

Fig..7 a) Detection of vague scaling b) Vague Skin Image

\section{F. Growth Rate Estimation And Classification}

A multivariate logistic regression was estimated to identify factors associated with a higher likelihood of image. The intensity measure of the sample image under study is calculated to predict and estimate the growth rate of the disease. The estimated growth rate is compared with other samples so as to classify and diagnose the sample accurately. A graph is generated that depicts the type of psoriasis either plague or erythrodermic.

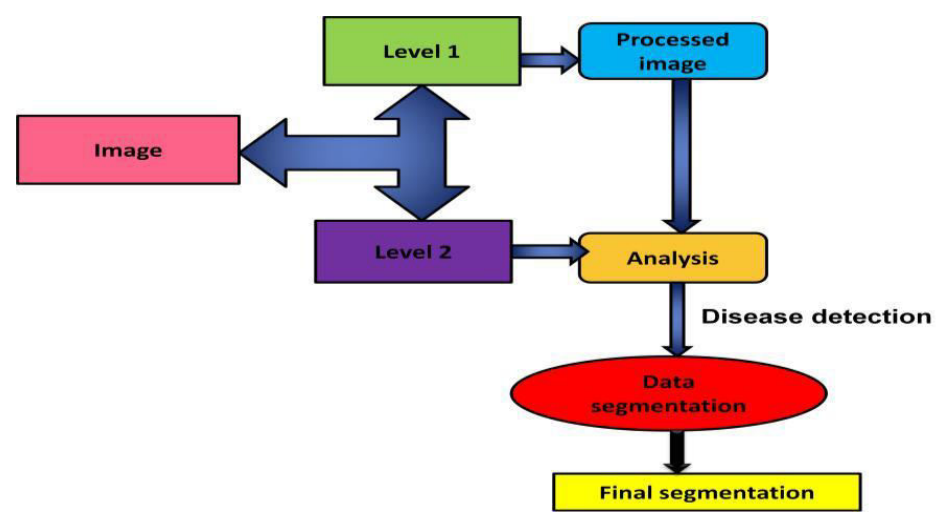

Fig. 8 Growth rate estimation 


\section{EXPERIMENTAL RESULTS}

The input images were taken under varying environmental conditions. The size of the image can be of any dimensions.

This system is designed to predict the type of psoriasis, especially for plague psoriasis and erythrodermic psoriasis. The execution speed is found to be higher for the proposed system than the existing system. This result is shown with the help of bar graph. The type of psoriasis is concluded based on the possibility of occurrence between the two types. The algorithm used here works well even for skin with wrinkles, moles and hairs. The execution speed is different for different images.

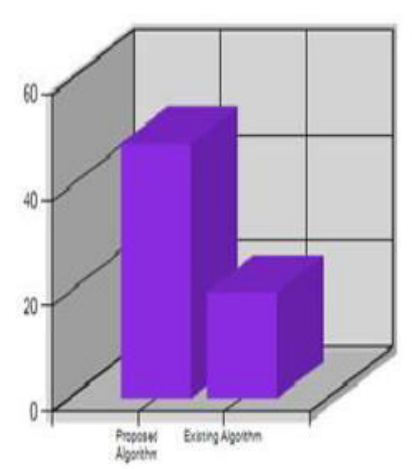

merisurance Manipulabson

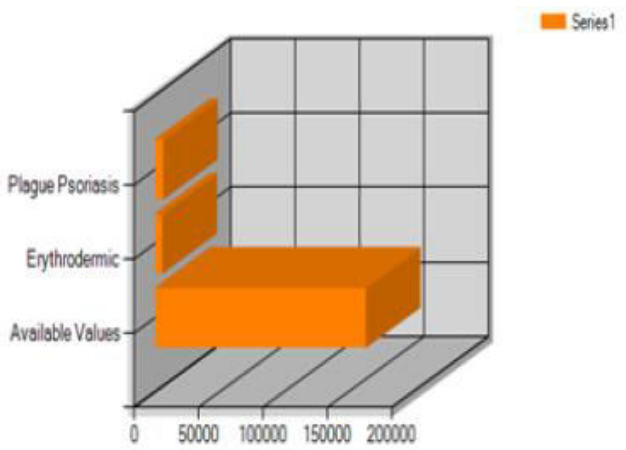

bertied Dsease Page Prorass

Fig.9 a) Performance evaluation b) Type of psoriasis

\section{CONCLUSION}

In this paper, the growth rate estimation of the psoriasis is detected. The results are reliable as it does not suffer from inter- and intra-observer differences. This paper involves four stages. In the first step, we perform the feature extraction which involves the color and texture analysis using Gabor filters. It includes the removal of erythema pixels and prepares a feature space image by removing the moles, hairs and other unwanted pixels from the consideration. The next step is the classification process which separates the scaling pixels and the normal skin pixels from the psoriatic image using SVM \& MRF. We make use of the soft constrained K-means clustering algorithm to obtain the training data to be used in SVM classification. The third stage is the vague skin detection which makes use of the parameter sigma to obtain the MRF segmentation. Finally using this vague skin image, the growth rate of the affected area in the psoriatic lesion is estimated and it also identifies the type of psoriasis.

Even though our algorithm works for varying size of images, it works efficiently for the images within the range between $940 \times 666$ and $161 \times 142$. In future we will try to develop algorithms to detect the other types of psoriasis such as guttate psoriasis, pustular psoriasis etc.

\section{REFERENCES}

[1] K. Busse and M. John Koo, "Residents' reports: Goeckerman combination therapy with low-dose acitretin for $\mathrm{HCV}$-associated psoriasis," Practical Dermatol., pp. 25-26, Apr. 2010.

[2] M.Meier and P.B. Sheth, "Clinical spectrumand severity of psoriasis," Curr. Probl. Dermatol., vol. 38, pp. 1-20, 2009.

[3] R. G. Langley and C. N. Ellis, "Evaluating psoriasis with psoriasis area and severity index, psoriasis global assessment, and lattice system physician's global assessment," J. Am. Acad. Dermatol., vol. 51, no. 4, pp. 563-569, 2004.

[4] J. Taur, G. Lee, C. Tao, C. Chen, and C. Yang, "Segmentation of psoriasis vulgaris images using multiresolution-based orthogonal subspace techniques," IEEE Trans. Syst.,Man, Cybernet., Part B: Cybernet., vol. 36, no. 2, pp. 390-402, Apr. 2006.

[5] D. Delgado, B. Ersboll, and J.M. Carstensen, "An image based system to automatically and objectively score the degree of redness and scaling in psoriasis lesions," in Proc. 13th Danish Conf. Image Anal. Pattern Recognit., 2004, pp. 130-137. 